\title{
Blood glucose level normalization and accurate timing improves the accuracy of PET-based treatment response predictions in rectal cancer
}

Citation for published version (APA):

Janssen, M. H. M., Ollers, M. C., van Stiphout, R. G. P. M., Riedl, R. G., van den Bogaard, J., Buijsen, J., Lambin, P., \& Lammering, G. (2010). Blood glucose level normalization and accurate timing improves the accuracy of PET-based treatment response predictions in rectal cancer. Radiotherapy and Oncology, 95(2), 203-208. https://doi.org/10.1016/j.radonc.2010.01.021

Document status and date:

Published: 01/05/2010

DOI:

10.1016/j.radonc.2010.01.021

Document Version:

Publisher's PDF, also known as Version of record

Document license:

Taverne

Please check the document version of this publication:

- A submitted manuscript is the version of the article upon submission and before peer-review. There can be important differences between the submitted version and the official published version of record.

People interested in the research are advised to contact the author for the final version of the publication, or visit the DOI to the publisher's website.

- The final author version and the galley proof are versions of the publication after peer review.

- The final published version features the final layout of the paper including the volume, issue and page numbers.

Link to publication

\footnotetext{
General rights rights.

- You may freely distribute the URL identifying the publication in the public portal. please follow below link for the End User Agreement:

www.umlib.nl/taverne-license

Take down policy

If you believe that this document breaches copyright please contact us at:

repository@maastrichtuniversity.nl

providing details and we will investigate your claim.
}

Copyright and moral rights for the publications made accessible in the public portal are retained by the authors and/or other copyright owners and it is a condition of accessing publications that users recognise and abide by the legal requirements associated with these

- Users may download and print one copy of any publication from the public portal for the purpose of private study or research.

- You may not further distribute the material or use it for any profit-making activity or commercial gain

If the publication is distributed under the terms of Article 25fa of the Dutch Copyright Act, indicated by the "Taverne" license above, 
PET in rectal cancer

\title{
Blood glucose level normalization and accurate timing improves the accuracy of PET-based treatment response predictions in rectal cancer
}

\author{
Marco H.M. Janssen ${ }^{\mathrm{a},{ }^{*},}$ Michel C. Öllers ${ }^{\mathrm{a}}$, Ruud G.P.M. van Stiphout ${ }^{\mathrm{a}}$, Robert G. Riedl ${ }^{\mathrm{b}}$, \\ Jørgen van den Bogaard ${ }^{a}$, Jeroen Buijsen ${ }^{a}$, Philippe Lambin ${ }^{\text {a,1 }}$, Guido Lammering ${ }^{\text {a,1 }}$ \\ ${ }^{a}$ Department of Radiation Oncology (MAASTRO), GROW Research Institute, University Medical Centre Maastricht, Maastricht, The Netherlands; ${ }^{b}$ Department of Pathology, \\ University Medical Centre Maastricht, Maastricht, The Netherlands
}

\section{A R T I C L E I N F O}

Article history:

Received 2 October 2009

Received in revised form 8 January 2010

Accepted 28 January 2010

Available online 20 February 2010

\section{Keywords:}

Blood glucose level normalization

Sequential FDG-PET-CT imaging

Response prediction

Rectal cancer

\begin{abstract}
A B S T R A C T
Purpose: To quantify the influence of fluctuating blood glucose level (BGLs) and the timing of PET acquisition on PET-based predictions of the pathological treatment response in rectal cancer.

Material and methods: Thirty patients, diagnosed with locally advanced-rectal-cancer (LARC), were included in this prospective study. Sequential FDG-PET-CT investigations were performed at four time points during and after pre-operative radiochemotherapy (RCT). All PET-data were normalized for the BGL measured shortly before FDG injection. The metabolic treatment response of the tumor was correlated with the pathological treatment response.

Results: During RCT, strong intra-patient BGL-fluctuations were observed, ranging from -38.7 to $95.6 \%$. BGL-normalization of the SUVs revealed differences ranging from -54.7 to $34.7 \%(p<0.001)$. Also, a SUV $_{\max }$ time-dependency of $1.30 \pm 0.66$ every $10 \mathrm{~min}$ (range: $0.39-2.58$ ) was found during the first 60 min of acquisition. When correlating the percent reduction of SUV $\max$ after 2 weeks of RCT with the pathological treatment response, a significant increase $(p=0.027)$ in the area under the curve of ROC-curve analysis was found when normalizing the PET-data for the measured BGLs, indicating an increase of the predictive strength.

Conclusions: This study strongly underlines the necessity of BGL-normalization of PET-data and a precise time-management between FDG injection and the start of PET acquisition when using sequential FDG-PET-CT imaging for the prediction of pathological treatment response.
\end{abstract}

(C) 2010 Elsevier Ireland Ltd. All rights reserved. Radiotherapy and Oncology 95 (2010) 203-208
Sequential FDG-PET-CT imaging has emerged as a promising method for the prediction of treatment response during or after neo-adjuvant radiochemotherapy (RCT) in an increasing number of malignancies [1-17]. For rectal cancer, the (percent) reduction of the maximum standardized-uptake-value $\left(\mathrm{SUV}_{\max }\right)$ within the tumor has been shown to predict the histo-pathological tumor response to pre-operative RCT [1-6,9,12,14,15]. An early prediction of the pathological treatment response of the tumor might in the future enable response-guided treatment algorithms on the basis of early changes of FDG uptake within the tumor $[2,5,11,12,15]$. However, a diversity of factors is known to influence the results of PET-imaging [5,18-23]. For PET-based predictions of the pathologic tumor response to pre-operative treatment, it is important to make sure that no or as little as possible fluctuations occur for the factors known to influence the results of sequential PET-imaging [5]. Especially the time interval between FDG injection and the

\footnotetext{
* Corresponding author. Maastro Clinic, Dr. Tanslaan 12, 6201 BN Maastricht The Netherlands, Box 1588.

E-mail address: marco.janssen@maastro.nl (M.H.M. Janssen).

1 These authors are equally contributed.
}

start of PET acquisition as well as the patient's blood glucose level (BGL) at the time of PET-imaging strongly influence the SUVs [5,18-23]. Despite the known advantage of delayed FDG-PETimaging for the detection of malignant lesions, the majority of studies published involving sequential PET-imaging in rectal cancer started the PET acquisition within the first 60 min after FDG injection [1-4,12]. Hamberg et al. stated that if SUV measurements are performed at the plateau, more robust predictions of treatment response could be achieved [20]. Moreover, it was found that the BGL of the patient at the time of PET-imaging inverse proportionally affects FDG uptake $[18,22,23]$. However, until now, most of the studies published on sequentially performed FDG-PET-images in rectal cancer did not pay attention to intra-patient BGL-fluctuations or the absence of a plateau for FDG uptake when analysing the acquired PET-data $[1-4,7-9,12]$. Although the concept correcting PET-data for BGL-fluctuations is not entirely new, the application and importance of it for the evaluation of treatment response using sequential FDG-PET-imaging is often overlooked, especially by the radiation oncology community.

Thus, we felt that it is of particular importance to investigate and quantify the influence of intra-patient BGL-fluctuations on 
sequentially acquired PET-scans and the resulting prediction of pathological treatment response as well as the influence of fluctuations in the time interval between FDG injection and the start of PET acquisition of rectal cancer patients.

\section{Materials and methods}

\section{Patient characteristics}

Thirty patients diagnosed with non-metastasized locally advanced-rectal-cancer (LARC) were included in this study from which the clinical TN staging was evaluated on a pre-treatment magnetic resonance (MR) scan (Table 1). All patients were referred to pre-operative treatment with radiotherapy (28 fractions of $1.8 \mathrm{~Gy}$ daily) and concomitant chemotherapy (capecitabine $825 \mathrm{mg} / \mathrm{m}^{2}$ BID), followed by a total mesorectal excision (TME). According to the Dutch law, the medical ethics committee approved the trial. All patients gave written informed consent before entering the study.

\section{PET-CT acquisition and processing}

As a part of the study, all patients underwent sequential FDGPET-CT imaging at four different time points: prior to therapy, at the end of the first and second week of RCT and once shortly before surgery (Fig. 1). All PET-CT scans were performed as described in earlier work [24].

All patients were instructed to fast for at least $6 \mathrm{~h}$ prior to FDG injection. The patients were positioned equal to the radiotherapy treatment position using a laser alignment system to have minimal variations between imaging and treatment conditions and between the different imaging time points. For the PET-CT scan, an intravenous injection of FDG (weight $(\mathrm{kg}) * 4+20 \mathrm{MBq}$ ) was performed. The list-mode PET-data were reconstructed into 28 time-frames: $10 \times 30 \mathrm{~s}, 5 \times 60 \mathrm{~s}, 5 \times 120 \mathrm{~s}$ and $8 \times 300 \mathrm{~s}$. Addition-

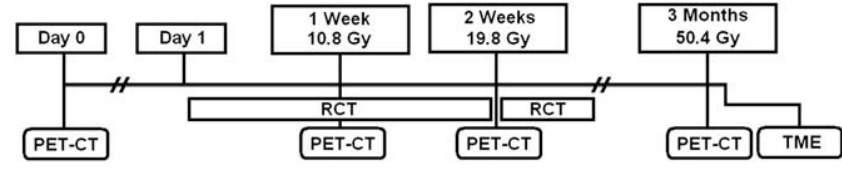

Fig. 1. Study scheme for the assessment of the metabolic treatment response for rectal cancer patients referred to pre-operative treatment with neo-adjuvant radiochemotherapy (RCT), followed by a total mesorectal excision (TME).

ally, the dynamic PET-data were corrected for tumor motion during dynamic acquisition, using the Image-Fusion-toolbox of the PMOD software package (PMOD Technologies Ltd., Zurich, Switzerland). Due to technical problems or patient incompliance, not all PET-CT scans could be performed as planned. For one patient no FDG could be injected for the PET-CT scan after 1 week of RCT, whereas two more patients refused PET-CT imaging after the second week of RCT. For six of the included patients, no PETCT scan could be performed prior to surgery.

\section{Blood-glucose levels}

Prior to each FDG injection, the patient's BGL was measured using an automatic device (LifeScan One Touch Ultra, LifeScan Inc., Milpitas, USA) [25]. All acquired PET-data were normalized for the BGL using the following equation:

$\mathrm{SUV}_{\text {normalized }}=\operatorname{SUV} \cdot \frac{(\mathrm{Glu})}{100}$,

with (Glu) the measured BGL (mg/dl) $[21,26]$.

\section{PET analysis}

For all PET-scans, the tumor was delineated using automated SUV-thresholding with the threshold (percentage of SUV $\mathrm{max}_{\max }$ within

Table 1

Overview of the patient characteristics. Clinical staging (cTNM), the measured blood glucose level (BGL) and the tumor regression grade (TRG).

\begin{tabular}{|c|c|c|c|c|c|c|c|c|}
\hline \multirow[t]{2}{*}{ Pat. Nr. } & \multirow[t]{2}{*}{ Weight (kg) } & \multirow[t]{2}{*}{ Age (years) } & \multirow[t]{2}{*}{ cTNM } & \multicolumn{4}{|l|}{$\mathrm{BGL}(\mathrm{mmol} / \mathrm{l})$} & \multirow[t]{2}{*}{ TRG } \\
\hline & & & & Pre-treatment & 1 week & 2 weeks & Pre-surgical & \\
\hline 1 & 75 & 48 & T3N1M0 & 5.7 & 6.4 & & 6.4 & 3 \\
\hline 2 & 86 & 64 & T2N1M0 & 6.2 & & 6.5 & & 1 \\
\hline 3 & 60 & 70 & T3N2M0 & 5.9 & 6.2 & 5.8 & 6.3 & 3 \\
\hline 4 & 103 & 57 & T3N2M0 & 5.2 & 5.2 & 5.4 & & 2 \\
\hline 5 & 64 & 78 & T3N2M0 & 6.1 & 5.9 & & 5.2 & 3 \\
\hline 6 & 70 & 71 & T4N2M0 & 6.4 & 5.6 & 5.7 & 6.2 & 2 \\
\hline 7 & 79 & 83 & T3N1M0 & 6.7 & 4.5 & 6.4 & 6.3 & 2 \\
\hline 8 & 83 & 60 & T3N2M0 & 5.5 & 5.3 & 5.8 & 4.9 & 2 \\
\hline 9 & 75 & 71 & T3N2M0 & 6.3 & 6.9 & 6.7 & 6.6 & 3 \\
\hline 10 & 84 & 66 & T3N1M0 & 10.6 & 7.9 & 8.1 & 6.5 & 3 \\
\hline 11 & 70 & 71 & T3N2M0 & 5.9 & 6.6 & 7.1 & 6.7 & 3 \\
\hline 12 & 69 & 60 & T3N2M0 & 6.4 & 5.4 & 6.0 & 6.7 & 4 \\
\hline 13 & 70 & 73 & T3N1M0 & 5.4 & 5.6 & 6.4 & & 1 \\
\hline 14 & 84 & 64 & T3N2M0 & 6.3 & 6.1 & 6.4 & 5.5 & 4 \\
\hline 15 & 102 & 54 & T3N1M0 & 5.4 & 5.3 & 5.3 & 5.3 & 3 \\
\hline 16 & 55 & 45 & T3NOMO & 5.3 & 5.9 & 5.8 & 5.8 & 3 \\
\hline 17 & 62 & 61 & T3N2M0 & 5.3 & 5.1 & 5.0 & & 3 \\
\hline 18 & 79 & 69 & T3N1M0 & 6.4 & 6.6 & 6.7 & & 2 \\
\hline 19 & 90 & 71 & T3N2M0 & 5.4 & 5.9 & 5.9 & 6.3 & 3 \\
\hline 20 & 88 & 70 & T3N2M0 & 6.1 & 6.4 & 6.1 & 7.8 & 4 \\
\hline 21 & 69 & 55 & T3N2M0 & 7.2 & 8.7 & 8.3 & 7.1 & 1 \\
\hline 22 & 75 & 74 & T3N2M0 & 6.2 & 6.8 & 6.2 & 5.5 & 2 \\
\hline 23 & 68 & 71 & T3NOMO & 5.7 & 5.9 & 6.5 & 6.6 & 4 \\
\hline 24 & 83 & 69 & T3N2M1 & 6.8 & 11.8 & 12.2 & 13.3 & 3 \\
\hline 25 & 52 & 77 & T4N1M0 & 5.3 & 7.6 & 7.7 & 6.8 & 4 \\
\hline 26 & 79 & 73 & T3NOMO & 5.2 & 5.4 & 5.7 & 4.9 & 2 \\
\hline 27 & 70 & 76 & T3NOMO & 5.7 & 6.2 & 6.7 & 5.9 & 1 \\
\hline 28 & 84 & 66 & T3N2M0 & 6.2 & 6.7 & 6.4 & 6.1 & 2 \\
\hline 29 & 98 & 52 & T3N2M0 & 8.6 & 6.5 & 5.8 & & 2 \\
\hline 30 & 110 & 43 & T3N1M0 & 6.3 & 6.1 & 6.1 & 6.7 & 4 \\
\hline
\end{tabular}


the tumor) depending on the tumor-to-background signal ratio (SBR-method), with the gluteus muscle selected as relevant background $[27,28]$. Dedicated software (TrueD VC50, Siemens Medical, Erlangen, Germany) was used for the quantification of FDG uptake within the tumor. Subsequently, response indices (RIs), indicating the percentage reduction of the FDG uptake relative to the pretreatment-measured value, were calculated for all three followup FDG-PET-CT scans. If no residual metabolic activity was 2present on the pre-surgical PET-scan, the patient was classified as a metabolic complete responder ( $\mathrm{mCR}$ ) and $\mathrm{SUV}_{\max }$ and the RI were set to respectively 0 and $100 \%$ for the pre-surgical PET-data.

\section{PET acquisition timing}

To study the time-dependency of SUV $\mathrm{max}_{\text {max }}$ determinations within the tumor, a tumor contour was generated on the last time frame of the dynamic PET-data using dedicated software (PMOD Technologies Ltd., Zurich, Switzerland). The maximum time-activity-curve (TAC) of the tumor was visually inspected for the presence of a decrease in the FDG uptake rate during the first 60 min after FDG injection indicating saturation for FDG uptake. For each of the included patients, the time dependencies of the $S_{U V} V_{\max }$ determination were calculated over the last eight time-frames ( $40 \mathrm{~min}$ ) of the maximum TAC and expressed as the difference in $\mathrm{SUV}_{\max }$ resulting from a 10 min time discrepancy between FDG injection and PET acquisition. Ten of the included patients underwent two additional static PET-CT scans prior to the start of therapy after FDG uptake periods of respectively 90 and $120 \mathrm{~min}$. The maximum TAC resulting from dynamic PET acquisition as well as the SUVs $s_{\max }$ calculated from the two additional static PET-scans were fitted into combined TACs over $120 \mathrm{~min}$.

\section{Pathological tumor response}

After the TME, the pathological treatment response within the tumor was evaluated by determination of the tumor regression grade (TRG) according to the Mandard-criteria: TRG1, complete tumor response; TRG2, residual cancer cells scattered through fibrosis; TRG3, an increased number of residual cancer cells, with predominant fibrosis; TRG4, residual cancer outgrowing fibrosis; TRG5, no regressive changes within the tumor [29]. All tumors were retrospectively classified by an experienced pathologist (RR), who was blinded for the PET-data. Based on the TRGs, the tumors were grouped into two groups, respectively pathological responders (TRG1, 2) and non-responders (TRG3-5).

\section{Statistical analysis}

Statistical analyses were performed using SPSS (version 15.0; SPSS Inc., Chicago, IL, USA). Comparisons of related measurements were performed using a Wilcoxon-signed rank test, whereas a Mann-Whitney $U$ test was used in case of independent samples. Differences were considered to be significant when the $p$-value was less than 0.05. To study the influence of BGL-fluctuations on PET-based predictions of pathological treatment response, the RI of SUV $V_{\text {max }}$, both before and after BGL-normalization were correlated to the pathological treatment response using receiver operating characteristics (ROC) analysis. A $z$-test was used to study the statistical significance of the difference between the ROC-curves before and after BGL-normalization [30].

\section{Results}

\section{Intra-patient BGL-fluctuations}

For the included patients, large intra-patient BGL-fluctuations were observed, ranging from -38.7 to $95.6 \%$ (average: $5.2 \pm 20.9 \%$ ) when compared to the pre-treatment-measured BGL (Table 1). BGL-normalization of the sequential PET-data resulted in an average increase of $S_{U V} V_{\max }$ of $9.7 \pm 14.1 \%$ (range: -54.7 to $34.7 \%$ ) when comparing the maximum SUVs before and after BGL-normalization

Table 2

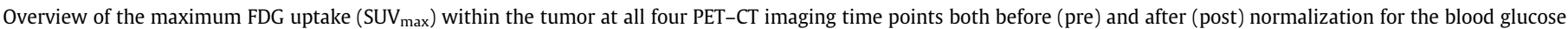
level measured shortly before FDG injection.

\begin{tabular}{|c|c|c|c|c|c|c|c|c|}
\hline \multirow[t]{2}{*}{ Pat. Nr. } & \multicolumn{2}{|c|}{ SUV $_{\max }$ pre-treatment (-) } & \multicolumn{2}{|c|}{$\mathrm{SUV}_{\max } 1$ week (-) } & \multicolumn{2}{|c|}{ SUV $_{\max } 2$ weeks (-) } & \multicolumn{2}{|c|}{ SUV $_{\max }$ pre-surgical (-) } \\
\hline & Pre & Post & Pre & Post & Pre & Post & Pre & Post \\
\hline 1 & 15.6 & 16.0 & 9.2 & 10.6 & & & 10.1 & 11.6 \\
\hline 2 & 11.9 & 13.3 & & & 5.5 & 6.4 & & \\
\hline 3 & 12.4 & 13.2 & 12.6 & 14.1 & 7.4 & 7.7 & 0.0 & 0.0 \\
\hline 4 & 20.9 & 19.6 & 10.9 & 10.2 & 6.2 & 6.0 & & \\
\hline 5 & 15.3 & 15.6 & 11.2 & 10.3 & & & 6.2 & 5.9 \\
\hline 6 & 12.5 & 14.4 & 10.5 & 10.6 & 8.6 & 8.8 & 6.5 & 6.5 \\
\hline 7 & 23.3 & 28.1 & 20.9 & 16.9 & 8.6 & 9.9 & 0.0 & 0.0 \\
\hline 8 & 8.4 & 8.3 & 8.5 & 8.1 & 8.1 & 8.5 & 6.9 & 6.1 \\
\hline 9 & 12.6 & 14.3 & 9.9 & 12.3 & 8.1 & 9.8 & 8.1 & 8.3 \\
\hline 10 & 8.8 & 16.9 & 10.9 & 15.5 & 12.9 & 18.9 & 6.6 & 7.8 \\
\hline 11 & 19.6 & 20.8 & 10.1 & 12.0 & 8.5 & 10.9 & 4.2 & 5.1 \\
\hline 12 & 9.6 & 11.1 & 10.2 & 10.0 & 8.8 & 9.5 & 7.3 & 8.8 \\
\hline 13 & 20.9 & 20.3 & 10.8 & 10.9 & 5.2 & 6.0 & & \\
\hline 14 & 11.0 & 12.5 & 8.9 & 9.8 & 7.7 & 8.9 & 6.6 & 6.5 \\
\hline 15 & 26.7 & 26.7 & 23.8 & 23.8 & 16.1 & 16.1 & 0.0 & 0.0 \\
\hline 16 & 7.8 & 7.4 & 8.5 & 9.0 & 6.8 & 7.1 & 3.4 & 4.0 \\
\hline 17 & 28.9 & 27.6 & 29.8 & 27.4 & 17.7 & 17.7 & & \\
\hline 18 & 13.4 & 15.5 & 13 & 15.5 & 11.6 & 14.0 & & \\
\hline 19 & 15.8 & 15.2 & 13.3 & 14.1 & 9.5 & 10.1 & 0.0 & 0.0 \\
\hline 20 & 16.6 & 18.2 & 12.6 & 14.5 & 11.8 & 13.0 & 8.6 & 12.1 \\
\hline 21 & 20.2 & 26.2 & 12.7 & 19.9 & 8.0 & 11.9 & 5.2 & 5.7 \\
\hline 22 & 13.8 & 15.4 & 11.8 & 14.4 & 7.5 & 8.4 & 4.3 & 4.3 \\
\hline 23 & 11.2 & 11.5 & 11.9 & 12.7 & 9.3 & 10.9 & 4.4 & 5.2 \\
\hline 24 & 8.0 & 9.8 & 5.5 & 11.7 & 4.8 & 10.6 & 0.0 & 0.0 \\
\hline 25 & 10.6 & 10.2 & 7.4 & 10.1 & 8.5 & 11.8 & 7.5 & 9.1 \\
\hline 26 & 15.5 & 14.2 & 9.9 & 8.1 & 9.8 & 7.3 & 4.1 & 3.6 \\
\hline 27 & 17.2 & 17.7 & 8.6 & 9.6 & 4.6 & 5.6 & 0.0 & 0.0 \\
\hline 28 & 21.4 & 23.9 & 12.2 & 14.7 & 11.3 & 13.0 & 7.6 & 8.4 \\
\hline 29 & 15.9 & 24.6 & 14.6 & 17.1 & 12.5 & 13.1 & & \\
\hline 30 & 9.9 & 11.2 & 8.8 & 9.7 & 11 & 12.0 & 6.2 & 7.4 \\
\hline
\end{tabular}




\section{A}

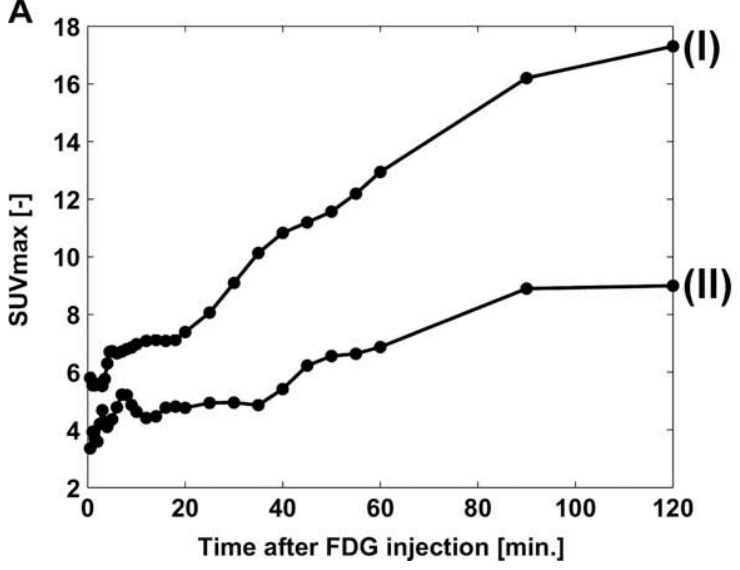

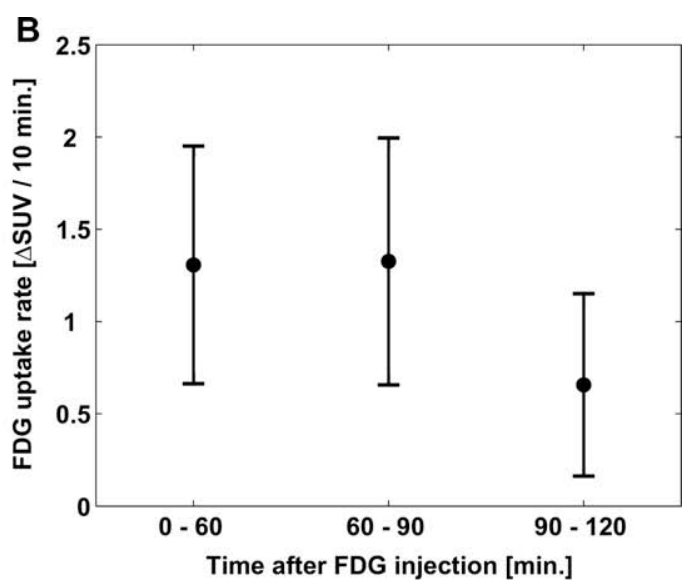

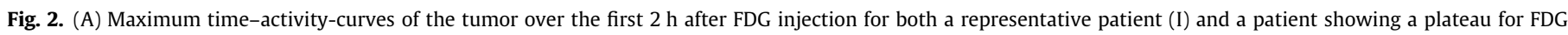

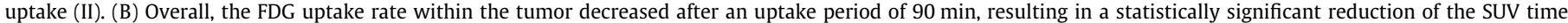
sensitivity $(p=0.005)$.

$(p<0.001)$ (Table 2). Large individual differences were noticed in the magnitude of the intra-patient BGL-fluctuations, leading to remarkable changes in the time trends of FDG uptake when applying BGLnormalization for some patients, whereas for other patients almost no fluctuations of the BGL were observed (Tables 1 and 2).

\section{SUV time sensitivity}

Visual analysis of the maximum TACs of the tumor revealed that in none of the investigated tumors a plateau for FDG uptake occurred within the first 60 min after FDG injection. Importantly, the determination of the $\mathrm{SUV}_{\max }$ within the first $60 \mathrm{~min}$ after FDG injection emerged as very time sensitive leading to large variations in the values with an average difference of $1.30 \pm 0.66$ in only $10 \mathrm{~min}$ (range: 0.39-2.58 $\Delta \mathrm{SUV}_{\max } / 10 \mathrm{~min}$ ). Only one of the investigated tumors reached a plateau for FDG uptake within the first 120 min after FDG injection (Fig. 2). However, overall the FDG uptake rate started to decrease after an uptake period of $90 \mathrm{~min}$, resulting in a statistically significant reduction of the $\mathrm{SUV}_{\max }$ time sensitivity by $56.3 \pm 30.6 \%$ (range: 2.5 to $95.0 \%)(p=0.005)$ when compared to the first 60 min after FDG injection (Fig. 2).

\section{Metabolic and pathological response evaluation}

When quantifying the FDG uptake within the tumor, for each patient the highest FDG uptake was detected on the pre-treatment PET-CT scan, followed by a statistically significant reduction of $\mathrm{SUV}_{\max }$ during pre-operative RCT. From the pathological response evaluation, thirteen of the included patients (43\%) were classified as pathological responders (4 TRG1, 9 TRG2), whereas 17 patients (57\%) were classified as pathological non-responders (11 TRG3, 6 TRG4) (Table 1).

\section{Correlation between the metabolic and pathological treatment response}

When correlating the RIs of SUV $\mathrm{max}_{\text {max }}$ both before and after BGLnormalization to the pathological treatment response, statistically significant higher RIs of SUV $\max$ were found for the pathological responders after 2 weeks of RCT before BGL-normalization and after both 1 and 2 weeks of RCT after BGL-normalization (Fig. 3). When looking at the resulting $p$-values, it can be concluded that normalizing the sequential PET-data for the BGL of the patient at the time of PET-imaging improves the statistical significance of the difference between the RIs of SUV $\mathrm{V}_{\max }$ of pathological respond-

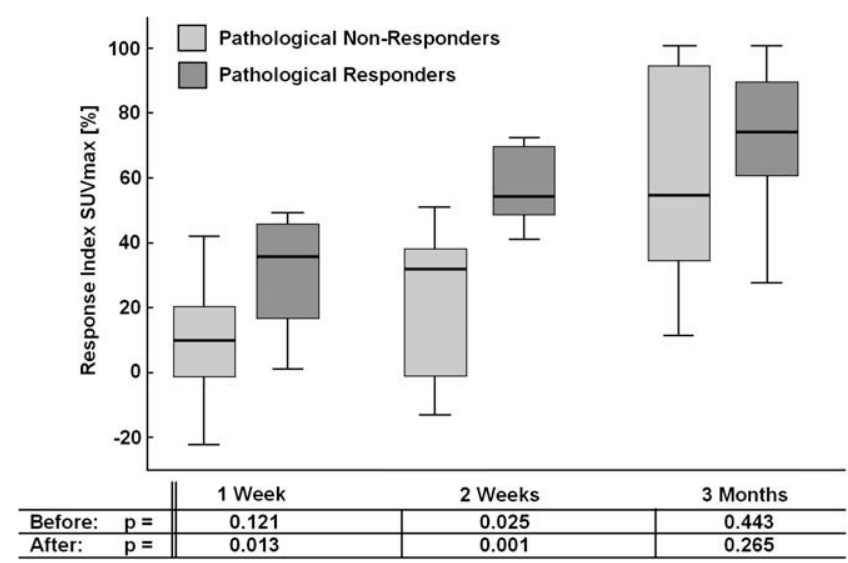

Fig. 3. Boxplots of the response indices of $S_{U V} V_{\max }$ measured during and after preoperative treatment for both pathological responders (dark boxes) and pathological non-responders (light boxes). The $p$-values indicate the statistical significance of the differences between the RIs of pathological responders and non-responders before and after blood glucose level normalization.

ers and non-responders (Fig. 3). ROC-curve analysis of the RI of $\mathrm{SUV}_{\max }$ after 2 weeks of pre-operative RCT presented an increase of the area under the curve (AUC) of the ROC-curve, from $0.749 \pm 0.097$ to $0.867 \pm 0.074(p=0.027)$, by performing a BGLnormalization of the PET-data, which proves a statistically significant increase in the accuracy of the prediction of pathological treatment response based on the reduction of $S U V_{\text {max }}$ after 2 weeks of RCT (Fig. 4A). Also, when plotting the RIs of SUV $\max$ relative to the TRG's, less overlap between the RIs of the pathological responders and non-responders were found after BGL-normalization of the PET-data when compared to the situation before normalization of the PET-data (Fig. 4B, C).

\section{Discussion}

Over the last years, sequential FDG-PET-CT imaging has been shown to be of promise for the prediction of pathological treatment response in an increasing number of malignancies [1-3,5$13,15,16]$. However, a diversity of factors are known to influence the results of PET-imaging, for instance the used equipment and protocol, uptake time of the FDG and the patients BGL at the time of PET-imaging $[5,19,20]$. When performing sequential FDG-PETCT imaging within a single patient, especially when using the 
A

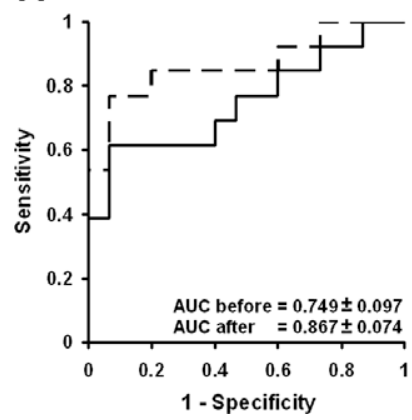

B

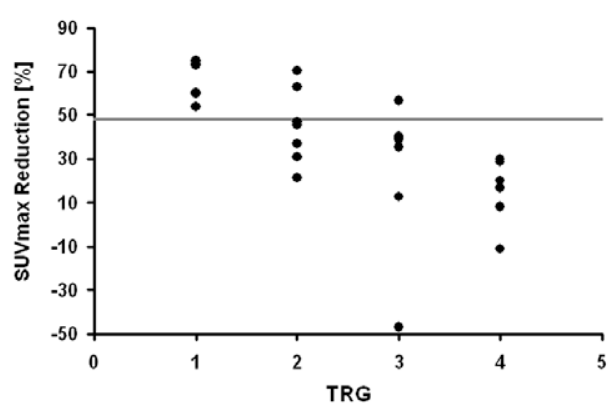

C After With BGL-normalization

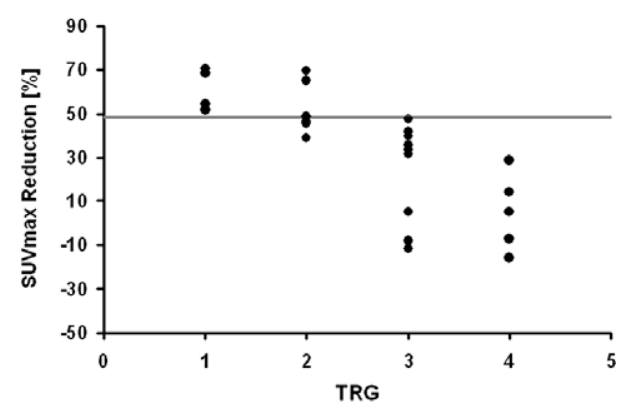

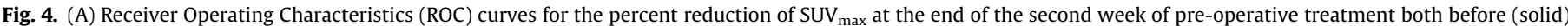

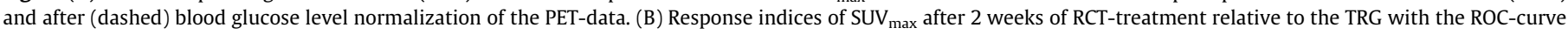

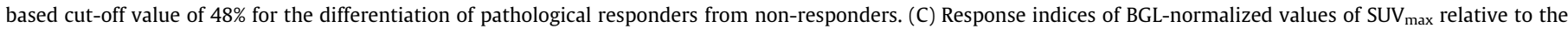
TRG with the same ROC-curve based cut-off value of $48 \%$ to differentiate between responders and non-responders.

SBR-method for tumor delineation, it is of high importance to use the same PET-CT scanner and imaging protocol for each of the examinations $[27,28]$. Analysis of the sequentially acquired PETdata without taking into account a BGL-normalization could, in the case of large intra-patient BGL-fluctuations, result in incorrect SUV time trends, which again could lead to an incorrect prediction of the treatment response. However, in earlier published literature, a clear trend was presented between the FDG uptake within the tumor and the BGL of the patients prior to FDG injection, enabling normalization of PET-data for the BGL of the patient measured shortly before FDG injection [22,23].

To the best of our knowledge, none of the published studies, except two, regarding treatment response predictions for rectal cancer based on sequential PET-imaging accounted for the BGL when evaluating the acquired PET-data $[10,15]$. For the patients included in our study, the SUVs normalized for the BGL were found to be statistically significant different from the SUVs before BGL-normalization, this in contrast to the findings of Stahl et al. [10]. Also, performing a normalization of the PET-data for the measured BGLs resulted in an increased AUC of ROC-curve analysis and a decreased $p$-value when predicting the pathological treatment response based on the percent reduction of $S_{U V} V_{\max }$ after 2 weeks of RCT. The significantly increased AUC of the ROC-curve after BGL-normalization as well as the decrease in overlap between responders and non-responders prove that performing a BGL-normalization for sequential PET-data improves the accuracy of the prediction of pathological treatment response.

Within this study, BGL measurements were performed using an automatic device as we did not include venous blood-sampling, which would enable clinical and analytical BGL measurements. However, the used automatic device (LifeScan One Touch, LifeScan Inc., Milpitas, USA) was proven to be the most accurate device, resulting in $100 \%$ of the measurements in the clinically accurate range for blood-glucose levels above $3.89 \mathrm{mmol} / \mathrm{l}$ [25].

As can be concluded from this study, BGL-normalization improves the accuracy of PET-based response predictions. However, when using PET-based response prediction models in clinical practice in the near future, patients presenting with large BGL-fluctuations or patients known to suffer from diabetes mellitus should be handled with care or even be excluded from further analysis to avoid erroneous response-guided modifications of the treatment protocol.

Another important finding of this study is the fact that the time interval between FDG injection and the start of PET acquisition in sequential FDG-PET-CT scans needs to be very consistent with the initially performed FDG-PET-CT scan, as FDG continues to show uptake by the tumor at high rates even 60 min after FDG injection. The determination of a SUV before saturation of FDG uptake within the tumor results in substantial SUV-variations if time differences between FDG injection and start of PET acquisition occur $[5,19,20]$. Although this SUV time sensitivity is the highest in the first 90 min after FDG injection, most protocols published involving the prediction of treatment response based on sequential PET-imaging in rectal cancer used uptake periods ranging from 40 to 60 min $[1-4,12]$. This might result in substantial variations in SUV measurements leading to misinterpretations in pathological response predictions. Delayed PET acquisition after 90 min. However, significantly reduces this SUV time-dependency. Therefore, an accurate time-management between FDG injection and the start of PET acquisition with a consistent time interval for all sequentially performed FDG-PET-CT scans or a delayed start of the PET acquisition after $90 \mathrm{~min}$ is needed in order to minimize the time-dependency of the acquired SUV changes.

In conclusion, this study underlines the necessity for a strict protocol for sequential FDG-PET-CT imaging of rectal cancer patients, in which the blood glucose level should be determined and normalized for and consistent time intervals between FDG injection and the start of PET-imaging should be followed. Otherwise, strategies to differentiate between pathological responding and non-responding malignancies on the basis of absolute cut-off values for the (percent) reduction of FDG uptake should be handled with care.

\section{Conflict of interest notification}

We are not aware of any actual or potential conflicts of interest.

\section{Acknowledgements}

The authors would like to thank Siemens MI for technical and financial support. We acknowledge financial support from the Netherlands Organization for Health Research and Development (ZonMw; clinical fellowship awarded to G.L.).

\section{References}

[1] Kalff V, Duong C, Drummond EG, Matthews JP, Hicks RJ. Findings on 18F-FDG PET scans after neoadjuvant chemoradiation provides prognostic stratification in patients with locally advanced rectal carcinoma subsequently treated by radical surgery. J Nucl Med 2006;47:14-22.

[2] Cascini GL, Avallone A, Delrio P, et al. 18F-FDG PET is an early predictor of pathologic tumor response to preoperative radiochemotherapy in locally advanced rectal cancer. J Nucl Med 2006;47:1241-8.

[3] Capirci C, Rampin L, Erba PA, et al. Sequential FDG-PET/CT reliably predicts response of locally advanced rectal cancer to neo-adjuvant chemo-radiation therapy. Eur J Nucl Med Mol Imaging 2007;34:1583-93. 
[4] Capirci C, Rubello D, Chierichetti F, et al. Long-term prognostic value of 18FFDG PET in patients with locally advanced rectal cancer previously treated with neoadjuvant radiochemotherapy. Am J Roentgenol 2006;187:W202-208.

[5] Hindie E, Hennequin C, Moretti JL. Predicting response to chemoradiotherapy in rectal and oesophageal cancer with 18F-FDG: prognostic value and possible role in patient management. Eur J Nucl Med Mol Imaging 2007;34:1576-82.

[6] Amthauer H, Denecke T, Rau B, et al. Response prediction by FDG-PET after neoadjuvant radiochemotherapy and combined regional hyperthermia of rectal cancer: correlation with endorectal ultrasound and histopathology. Eur J Nucl Med Mol Imaging 2004;31:811-9.

[7] Denecke T, Rau B, Hoffmann KT, et al. Comparison of CT, MRI and FDG-PET in response prediction of patients with locally advanced rectal cancer after multimodal preoperative therapy: is there a benefit in using functional imaging? Eur Radiol 2005;15:1658-66.

[8] Guillem JG, Moore HG, Akhurst T, et al. Sequential preoperative fluorodeoxyglucose-positron emission tomography assessment of response to preoperative chemoradiation: a means for determining longterm outcomes of rectal cancer. J Am Coll Surg 2004;199:1-7.

[9] Melton GB, Lavely WC, Jacene HA, et al. Efficacy of preoperative combined 18 fluorodeoxyglucose positron emission tomography and computed tomography for assessing primary rectal cancer response to neoadjuvant therapy. J Gastrointest Surg 2007;11:961-9 (discussion 969).

[10] Stahl A, Ott K, Schwaiger M, Weber WA. Comparison of different SUV-based methods for monitoring cytotoxic therapy with FDG PET. Eur J Nucl Med Mol Imaging 2004;31:1471-8.

[11] Wieder $\mathrm{HA}$ Ott $\mathrm{K}$, Lordick $\mathrm{F}$, et al. Prediction of tumor response by FDG-PET: comparison of the accuracy of single and sequential studies in patients with adenocarcinomas of the esophagogastric junction. Eur J Nucl Med Mol Imaging 2007;34:1925-32.

[12] Rosenberg R, Herrmann K, Gertler R, et al. The predictive value of metabolic response to preoperative radiochemotherapy in locally advanced rectal cancer measured by PET/CT. Int J Colorectal Dis 2009;24:191-200.

[13] van Baardwijk A, Bosmans G, Dekker A, et al. Time trends in the maximal uptake of FDG on PET scan during thoracic radiotherapy. A prospective study in locally advanced non-small cell lung cancer (NSCLC) patients. Radiother Oncol 2007;82:145-52.

[14] Vliegen RF, Beets-Tan RG, Vanhauten B, et al. Can an FDG-PET/CT predict tumor clearance of the mesorectal fascia after preoperative chemoradiation of locally advanced rectal cancer? Strahlenther Onkol 2008;184:457-64.

[15] Janssen $\mathrm{MH}$, Ollers MC, Riedl RG, et al. Accurate prediction of pathological rectal tumor response after two weeks of preoperative radiochemotherapy using (18)F-fluorodeoxyglucose-positron emission tomography-computed tomography imaging. Int J Radiat Oncol Biol Phys 2009.

[16] Young H, Baum R, Cremerius U, et al. Measurement of clinical and subclinical tumour response using [18F]-fluorodeoxyglucose and positron emission tomography: review and 1999 EORTC recommendations. European Organization for Research and Treatment of Cancer (EORTC) PET Study Group. Eur J Cancer 1999;35:1773-82.

[17] Monteil J, Mahmoudi N, Leobon S, et al. Chemotherapy response evaluation in metastatic colorectal cancer with FDG PET/CT and CT scans. Anticancer Res 2009;29:2563-8.

[18] Crippa F, Gavazzi C, Bozzetti F, et al. The influence of blood glucose levels on [18F]fluorodeoxyglucose (FDG) uptake in cancer: a PET study in liver metastases from colorectal carcinomas. Tumori 1997;83:748-52

[19] Keyes Jr JW. SUV: standard uptake or silly useless value? J Nucl Med 1995;36:1836-9.

[20] Hamberg LM, Hunter GJ, Alpert NM, Choi NC, Babich JW, Fischman AJ. The dose uptake ratio as an index of glucose metabolism: useful parameter or oversimplification? J Nucl Med 1994;35:1308-12.

21] Beaulieu S, Kinahan P, Tseng J, et al. SUV varies with time after injection in (18)F-FDG PET of breast cancer: characterization and method to adjust for time differences. J Nucl Med 2003;44:1044-50.

[22] Langen KJ, Braun U, Rota Kops E, et al. The influence of plasma glucose levels on fluorine-18-fluorodeoxyglucose uptake in bronchial carcinomas. J Nucl Med 1993;34:355-9.

[23] Lindholm P, Minn H, Leskinen-Kallio S, Bergman J, Ruotsalainen U, Joensuu H. Influence of the blood glucose concentration on FDG uptake in cancer - a PET study. J Nucl Med 1993;34:1-6.

[24] Janssen MH, Aerts HJ, Ollers MC, et al. Tumor delineation based on timeactivity curve differences assessed with dynamic fluorodeoxyglucose positron emission tomography-computed tomography in rectal cancer patients. Int J Radiat Oncol Biol Phys 2009;73:456-65.

[25] Brunner GA, Ellmerer M, Sendlhofer G, et al. Validation of home blood glucose meters with respect to clinical and analytical approaches. Diabetes Care 1998;21:585-90.

[26] Eary JF, Mankoff DA. Tumor metabolic rates in sarcoma using FDG PET. J Nucl Med 1998;39:250-4.

[27] Daisne JF, Sibomana M, Bol A, Doumont $T$, Lonneux M, Gregoire V. Tridimensional automatic segmentation of PET volumes based on measured source-to-background ratios: influence of reconstruction algorithms. Radiother Oncol 2003;69:247-50.

[28] Ollers M, Bosmans G, van Baardwijk A, et al. The integration of PET-CT scans from different hospitals into radiotherapy treatment planning. Radiother Oncol 2008;87:142-6.

[29] Mandard AM, Dalibard F, Mandard JC et al. Pathologic assessment of tumo regression after preoperative chemoradiotherapy of esophageal carcinoma. Clinicopathologic correlations. Cancer 1994;73:2680-6.

[30] Hanley JA, McNeil BJ. A method of comparing the areas under receiver operating characteristic curves derived from the same cases. Radiology $1983 ; 148: 839-43$ 